\title{
Sensitivity Analysis of a Car Shock Absorber Through a Functional Mock-up Units-Based Modelling Strategy
}

\author{
VUILLOD Bruno $^{1,2}$ HALlO Ludovic ${ }^{1} \quad$ PANETTIERI Enrico $^{2} \quad$ MONTEMURRO Marco $^{2}$
}

\author{
${ }^{1}$ Commissariat à l'énergie atomique et aux énergies alternatives (CEA), France, ludovic.hallo@cea.fr \\ ${ }^{2}$ Arts et Métiers Institute of Technology, Université de Bordeaux, CNRS, INRA, Bordeaux INP, HESAM Université, \\ I2M UMR 5295, F-33405 Talence, France, \{bruno.vuillod, enrico.panettieri, \\ marco.montemurro\}@ensam.eu
}

\begin{abstract}
In Model-Based System Engineering (MBSE), some functional sub-systems can have a considerable influence on the overall system behaviour, whilst the effect of other ones can be neglected. Of course, the former requires a refined modelling approach, whilst the latter can be suitably represented by means of low-fidelity models (usually OD models). Being capable of identifying the required precision level of sub-systems can help reducing the system complexity, with a negligible impact on the overall accuracy and help deepen the calculations in the system parts where it is necessary.

To determine which sub-systems models must be refined, suitable indicators must be introduced to assess their influence on the global system behaviour. To this purpose, in this work, a sensitivity analysis based on Sobol's indices coupled with a simple mechanical model developed in the Modelica environment is proposed to achieve the aforementioned task.
\end{abstract}

Keywords: Modelica, Sensitivity Analysis, Sobol's Index, Dynamical System.

\section{Introduction}

The first step to generate a Modelica model consists of setting the blocks scheme accounting for the global system architecture. The second step, is to enrich this scheme with additional components which include physical-based responses. This task may be difficult to be realised because it needs specific attention and a deep knowledge of multi-physics dynamical problems. Once the functional system architecture is finalised, Modelica can be used to solve it. This modelling approach, when complex systems are analysed, can lead to prohibitive computational costs, poor accuracy and incompatibility with project requirements, see, for instance, the different stages of a V-and-V organisation for example (Plogert 1996).

A viable strategy to improve the modelling approach, i.e. by proposing an efficient model constituting a good balance between accuracy and computational costs, consists in identifying those system parameters influencing the most the considered system response. This task is any- thing but trivial when using classical software. Modelica can be conveniently employed to achieve this task because it allows for splitting the whole system into sub-systems and allows identifying the main parameters influencing its overall behaviour.

In this paper, a model of a car shock absorber, based on the Functional Mock-up Units (FMU) file export option of Modelica, is developed and integrated in an in-house code which performs the sensitivity analysis. The car shock absorber is based on a classic mass-spring-damper model enhanced with some fundamental notions of hydromechanics. In particular, the proposed model is characterised by 11 input variables and a single output: the equilibrium position of the car shock absorber at the generic time. The interest of using FMU relies on fast multi-physics simulations, with the possibility to easily change the parameters set. Therefore, a statistical analysis (which requires a huge amount of simulation runs) can be carried out by modifying the parameters set through the use of FMU. The sensitivity analysis presented in this paper is based on the use of Sobol's indices (Sobol 1993), which allows for determining the model parameters influencing the most the considered output. Sobol's indices allows expressing the variance of a given output by accounting for the variance of each input variable and for the coupling effects among them (depending on the order of the Sobol's inde). This method is based on Hoeffding decomposition (Hoeffding 1948) and has been adapted to the discrete numerical systems by Saltelli (Saltelli 2002). The sensitivity analysis proposed in this work is carried out through an in-house code called Nebraska, coupled with the Modelica model via its FMU file and a Python code.

Only a few literature references exist on the sensitivity analysis based on Sobol's indices and on the Hoeffding decomposition: (Sobol 1993), (Hoeffding 1948), (Saltelli 2002). Nowadays, these approaches are being reconsidered with the development of parallel and/or distributed computing capabilities of modern computers, which enable a large number of runs in a reasonable time. Some recent works make use of Sobol's indices in different applications: global optimisation strategy based on metaheuristic algorithms (Janon 2019), metamodelling strategies (Marrel et al. 2009) or extension of the Sobol's index 
theory to the case of dependent input variables (Chastaing, Gamboa, and Prieur 2015).

The paper is organised as follows. In Section 2, the mathematical formulation at the basis of the proposed model is presented together with the fundamentals of the Sobol's analysis. In section 3, the details of the Modelica model are provided, whilst Section 4 presents the numerical results and the related discussion. Section 5 ends the paper with some concluding remarks and prospects.

Notation. Upper-case bold letters are used to indicate tensors and matrices, while lower-case bold letters indicate column vectors.

\section{System Description}

\subsection{The Mechanical System}

The mechanical system analysed in this work is a car shock absorber, illustrated in Figure 1. Four main elements can be identified within the CAD model: the piston is a cylinder with a central hole, which allows for the oil flow during motion. This allows also absorbing the spring oscillations. The external piston radius is considered as equal to the internal piston chamber radius, without gap. The passengers and the car masses are loaded on the previously assembly via the top mounting ring.

The scheme shown in Figure 1 represents the CAD system in functional diagram form: a spring-damper parallel system fixed to a support structure represents the car shock absorber submitted to the weight of the car and its passenger.

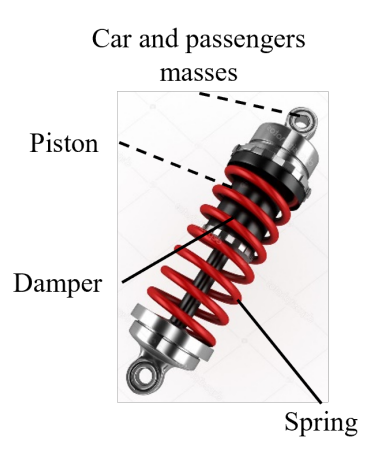

a)

$$
\text { b) }
$$

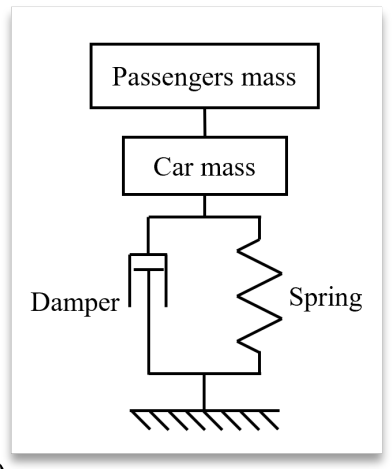

Figure 1. a) CAD model of the car shock absorber with b) its functional mechanical model.

The dynamic response of such system is governed by the longitudinal displacement $x(t)$ of the top chamber piston. It can be determined by solving the equilibrium equation of the system:

$$
M \mathbf{a}=\sum_{i=1}^{q} \mathbf{f}_{\mathrm{ext}, i},
$$

where $M=\frac{m_{P}}{4}+\frac{m_{C}}{4}$ is a quarter of the overall mass of the system (i.e. car + passengers masses), a the acceleration, whose component along the $\mathrm{x}$ axis, is equal to $\ddot{x}(t)$, $\mathbf{f}_{\mathrm{ext}, i}$ the generic $i$-th external force applied to the springdamper (see Figure 2) and $q$ their total number.

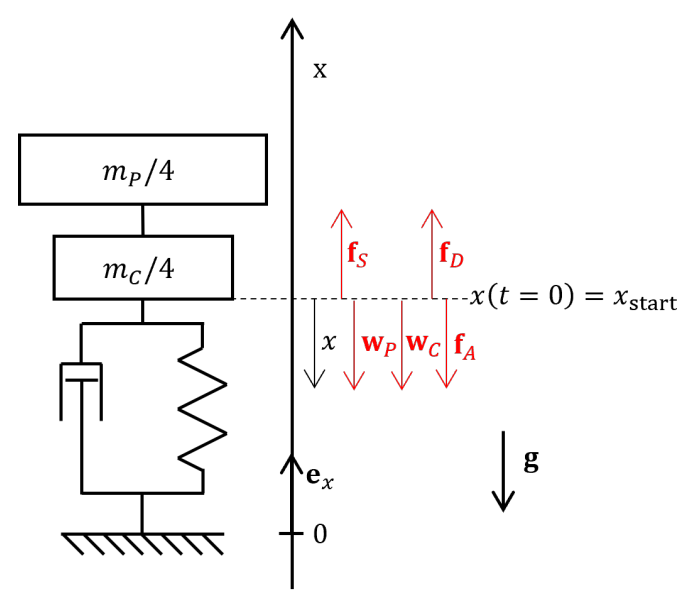

Figure 2. Functional scheme and external forces applied to a car shock absorber. The direction of the gravity acceleration is represented by the $\mathbf{g}$ vector.

The external forces applied to the spring-damper system are listed here below:

- Archimedes' force:

$$
\mathbf{f}_{A}=-S_{p} h \rho_{\text {oil }} g \mathbf{e}_{x},
$$

where $S_{p}=\pi\left(r_{\mathrm{ext}}^{2}-r_{\mathrm{int}}^{2}\right)$ is the cross-section area of the piston, $r_{\text {int }}$ and $r_{\text {ext }}$ are the internal and external piston radius, respectively, $h$ is the piston height, $\rho_{\text {oil }}$ is the oil density, $g$ is the gravity acceleration and $\mathbf{e}_{x}$ the unit vector of the $x$ axis. Note that the Archimedes' force is applied to the piston which will tend to rise in its chamber and exert a force in the direction of the vector $\mathbf{e}_{x}$.

- Damper force:

$$
\mathbf{f}_{D}=-\operatorname{sign}(\dot{x}) S_{p} \Delta P \mathbf{e}_{x},
$$

where $\Delta P$ is the pressure loss, computed by assuming the physical model shown in Figure 3. The value of $\Delta P$ is thus computed as follows (Idel'chik 1966):

$$
\Delta P=\rho_{\text {oil }} \frac{\omega_{0}^{2}}{2}\left(\zeta_{\text {red }}+\zeta_{\text {exp }}\right),
$$

where $\omega_{0}$ is the oil speed inside the piston. By imposing mass flux conservation, $\omega_{0}$ reads:

$$
\omega_{0}=\frac{\dot{x} S_{p}}{S_{h}}
$$

with $S_{h}=\pi r_{\text {int }}^{2}$ is the cross-section area of the piston hole. In addition, $\zeta_{\text {red }}$ and $\zeta_{\text {exp }}$ are expressed as follows: 


$$
\zeta_{\text {red }}=\left(\frac{1}{C}-1\right)^{2}, \zeta_{\exp }=\left(1-\frac{S_{h}}{S_{p}}\right)^{2}
$$

with $C=0.63+0.37\left(\frac{S_{h}}{S_{p}}\right)^{2}$.

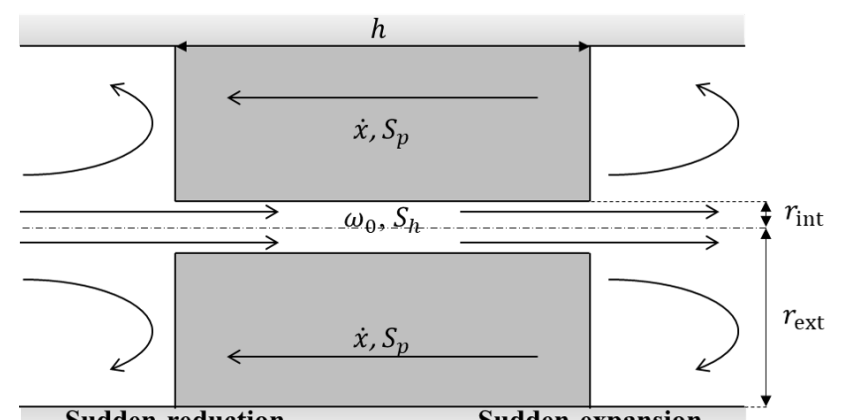

Sudden reduction

Sudden expansion

Figure 3. Different flows through the piston during its motion.

- Spring stress:

$$
\mathbf{f}_{S}=-k\left(x-L_{0}\right) \mathbf{e}_{x},
$$

where $k$ is the spring constant and $L_{0}$ its unstreched length. Note that, at initial time, the piston position is smaller than the unstreched spring length, i.e. the system is considered as pre-loaded.

- Weight action of the passengers:

$$
\mathbf{w}_{P}=-\frac{m_{P}}{4} g \mathbf{e}_{x},
$$

where $m_{P}$ is the overall passengers mass.

- Weight of the car:

$$
\mathbf{w}_{C}=-\frac{m_{C}}{4} g \mathbf{e}_{x},
$$

with $m_{C}$ the overall mass of the car.

\subsection{Sensitivity Analysis Based on Sobol's In- dices}

In this section, the fundamentals of the sensitivity analysis based on Sobol's indices (Sobol 1993) is briefly introduced. Sobol's indices allow for the identification of the input parameters having the stronger influence on the system outputs.

Consider a multiple-input-single-output (MISO) system whose transfer function is $\mathscr{M}: \xi \in \mathbb{R}^{n} \longrightarrow Y \in \mathbb{R}$, where $n$ is the number of inputs and $\mathscr{M}$ is represented by the physical model.

$\xi^{\mathrm{T}}=\left(\xi_{1}, \ldots, \xi_{n}\right)$ represents the vector of inputs. The variability of the inputs is modelled via random variables characterised by their probability density functions (PDFs): $\forall i \in\{1, \ldots, n\}, \xi_{i} \sim d P_{\xi_{i}}$ (which must be read: $\xi_{i}$ follows a distribution of $\mathrm{PDF} d P_{\xi_{i}}$ ). It is noteworthy that Sobol's indices can be defined either in the case of dependent variables or in the case of independent variables (Hoeffding 1948; Sobol 1993). In the following of this work, only independent inputs will be considered, i.e. the generic input variable cannot be expressed as a function of the remaining inputs (neither explicitly nor implicitly). This means that the probability measure of $\boldsymbol{\xi}$ is given by:

$$
d P_{\xi}=\prod_{i=1}^{n} d P_{\xi_{i}}
$$

The objective of the sensitivity analysis is to determine the relative influence of each parameter $\xi_{i}$, on the considered output value in term of variance. The Sobol's index $S_{i}$ of the variable $\xi_{i}$ gives the percentage of variance on the output associated solely to $\xi_{i}$. Similarly, $S_{i, j}$ gives the percentage of the output variance associated to the couple $\left(\xi_{i}, \xi_{j}\right)$ (and this concept must be extended to all possible $2^{n}+1$ combinations of input variables). Mathematically, the Sobol's index of order $1\left(S_{i}\right)$ and of order $2\left(S_{i, j}\right)$ can be expressed as follows:

$$
\begin{gathered}
S_{i}=\frac{\mathscr{V}_{i}}{\mathscr{V}}, \\
S_{i, j}=\frac{\mathscr{V}_{i, j}}{\mathscr{V}},
\end{gathered}
$$

with $\mathscr{V}_{i}$ the variance associated to $\xi_{i}, \mathscr{V}_{i, j}$ the variance associated to the couple $\left(\xi_{i}, \xi_{j}\right)$ and $\mathscr{V}$ the global variance associated to the observed variable $Y$ of the model $\mathscr{M}$. The above quantities are defined as follows:

$$
\mathscr{V}=\operatorname{Var}(\mathrm{E}(Y))
$$

$$
\begin{gathered}
\mathscr{V}_{i}=\operatorname{Var}\left(\mathrm{E}\left(Y \mid \xi_{i}\right)\right)-\operatorname{Var}(\mathrm{E}(Y)) \\
\mathscr{V}_{i, j}=\operatorname{Var}\left(\mathrm{E}\left(Y \mid \xi_{i}, \xi_{j}\right)\right)-\operatorname{Var}\left(\mathrm{E}\left(Y \mid \xi_{i}\right)\right) \\
-\operatorname{Var}\left(\mathrm{E}\left(Y \mid \xi_{j}\right)\right)+\operatorname{Var}(\mathrm{E}(Y))
\end{gathered}
$$

where $\mathrm{E}(Y)$ is the expected value of $Y, \mathrm{E}\left(Y \mid \xi_{i}\right)$, the conditional expected value of $Y$ regarding $\xi_{i}$ and $\mathrm{E}\left(Y \mid \xi_{i}, \xi_{j}\right)$ the conditional expected value of $Y$ regarding the pair of variables $\left(\xi_{i}, \xi_{j}\right)$. For a deeper insight in the matter, the reader is addressed to (Philippe and Viano 2010).

The $2^{n}+1$ Sobol indices can provide precious information for the sensitivity analysis, but their computation can be prohibitive when a large number of variables are considered. In (Saltelli 2002), the author presents a numerical integration scheme requiring $N(n+2)$ simulations, with $N$ the total number of samples allowing the computation of the $n$ elementary indices $\left(S_{i}\right)_{i \in[1, n]}$ together with the $n$ total indices defined by:

$$
\forall i \in\{1, \ldots, n\}, \mathrm{ST}_{i}=S_{i}+S_{i, j}+\ldots+S_{i, \ldots, n} .
$$


The total Sobol's indices give an indication of the influence of the variable $\xi_{i}$ on the considered output as an isolated variable and when $\xi_{i}$ is combined with every other set of variables. For example, if $\mathrm{ST}_{i} \approx 0$, the variable $\xi_{i}$ does not influence at all the considered output. Note that in the rest of the paper, the indices $S_{i}$ are indicated as $\mathrm{SE}_{i}$ and called elementary Sobol's indices. For a deeper insight in the matter the interested reader is addressed to (Héliot 2017-2018).

\section{Numerical Model of the Car Shock Absorber}

The mechanical model of the car shock absorber considered in this study is built through the Modelica programming language (Fritzson 2014) with the MapleSim software (Maplesoft 2014) and interfaced with the Nebraska code wherein sensitivity analysis by means of Sobol's indices is performed.

\subsection{Modelica Model}

To simulate the behaviour of the car shock absorber, the Modelica programming language has been employed, and, particularly, its capability to easily export model in FMU format.

The Modelica model is shown in Figure 4. The Modelica Standard Library (MSL) has been used to generate the majority of the components of the mechanical system. However, the mechanical behaviour of the damper has been modelled via a customized damper force element to reproduce the constitutive law of Eq. (3). It can be noticed that the element $M_{C}$ of Figure 4 (which represents a massless element with limited motion) has been introduced to limit the motion in the piston chamber (to ensure geometric/physical consistency). The weight action of the car is represented by the $W_{C}$ element, whilst the one of the passengers is represented by the $W_{P}$ element. The whole system is described by means of 11 parameters among which only six are considered for the sensitivity analysis.

When the car is loaded, its shock absorbers have the function to limit the spring oscillations and to dissipate them in a short time, at a given position. As a consequence, the output considered for the sensitivity analysis is the piston position at a given time $x(t)$. As a reference, the time constant for shock absorption has been set as $\tau_{\mathrm{abs}}=2 \mathrm{~s}$ with a stable piston position at $x=0.64 \mathrm{~m}$. The reference motion parameters are given in Table 1 and correspond approximately to a physically admissible situation. Note that the piston initial position is defined at $x_{\text {start }}=L_{\text {bar }}+L_{\text {cham }}$ with $L_{\text {bar }}$ the bag length in contact with the floor (assimilable to the wheel) and $L_{\text {cham }}$ the length of the piston chamber which limit the piston displacement. At $t=0 \mathrm{~s}$, the spring is preloaded because $x_{\text {start }}<\left(L_{0}+L_{\text {bar }}\right)$.

Once the Modelica model is created and validated, the FMU file is generated in co-simulation mode. The FMU

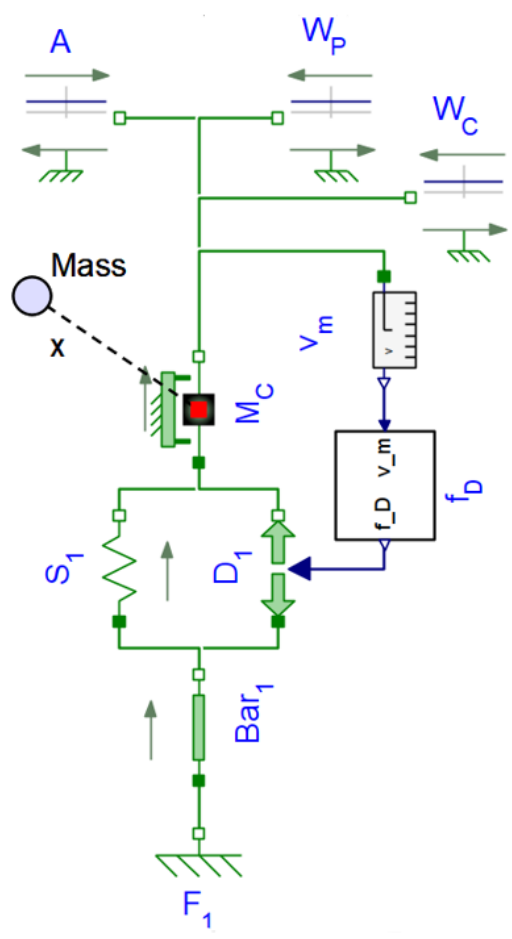

Figure 4. Modelica model of the car shock absorber.

Table 1. List of reference model parameters and their type.

\begin{tabular}{lll}
\hline Parameter & Reference value & Variable \\
\hline$k$ & $9000 \mathrm{~N} \cdot \mathrm{m}^{-1}$ & Yes \\
$L_{0}$ & $0.45 \mathrm{~m}$ & Yes \\
$r_{\text {int }}$ & $0.002 \mathrm{~m}$ & Yes \\
$r_{\text {ext }}$ & $0.03 \mathrm{~m}$ & No \\
$m_{P}$ & $150 \mathrm{~kg}$ & Yes \\
$h$ & $0.02 \mathrm{~m}$ & Yes \\
$\rho_{\text {oil }}$ & $884 \mathrm{~kg} \cdot \mathrm{m}^{-3}$ & No \\
$g$ & $9.81 \mathrm{~m} \cdot \mathrm{s}^{-2}$ & No \\
$L_{\text {bar }}$ & $0.5 \mathrm{~m}$ & No \\
$L_{\text {cham }}$ & $0.25 \mathrm{~m}$ & No \\
$m_{C}$ & $1000 \mathrm{~kg}$ & Yes \\
\hline
\end{tabular}

file of the mechanical system can be represented as a black box for which input parameters with their respective intervals of definition must be defined as reported in Table 2. These intervals, have been chosen according to admissible mechanical conditions of the shock absorbers. In particular, the interval of definition of the parameter $r_{\text {int }}$ is selected in order to ensure a complete or semi-periodic absorbing behaviour.

\subsection{Preliminary Analysis of the System}

Before proceeding with the sensitivity analysis, the Modelica model has been simplified. By evaluating the orders of magnitude of the external forces, the Archimedes' force can be neglected with respect to the spring force since its value differ by three orders of magnitude. As a conse- 
Table 2. List of the model input variables and the related interval.

\begin{tabular}{ll}
\hline Variable & Interval \\
\hline$k$ & {$[8000 ; 11000] \mathrm{N} \cdot \mathrm{m}^{-1}$} \\
$L_{0}$ & {$[0.4 ; 0.6] \mathrm{m}$} \\
$r_{\text {int }}$ & {$[0.001 ; 0.005] \mathrm{m}$} \\
$h$ & {$[0.01 ; 0.04] \mathrm{m}$} \\
$m_{P}$ & {$[60 ; 360] \mathrm{kg}$} \\
$m_{C}$ & {$[900 ; 1300] \mathrm{kg}$} \\
\hline
\end{tabular}

quence, the parameter $h$ can be removed from the input variables to be considered in the sensitivity analysis.

By applying this simplification, one can notice that, as expected, no significant variation occurs in the piston motion. The relative difference between the piston motion of the complete Modelica model and the counterpart of the simplified model vs. the time is illustrated in Figure 5. This difference is about $10^{-5} \mathrm{~m}$, which represent $0.008 \%$ of the stabilised motion reference (at $x=0.64 \mathrm{~m}$ ), thus the two models can be considered as equivalent.

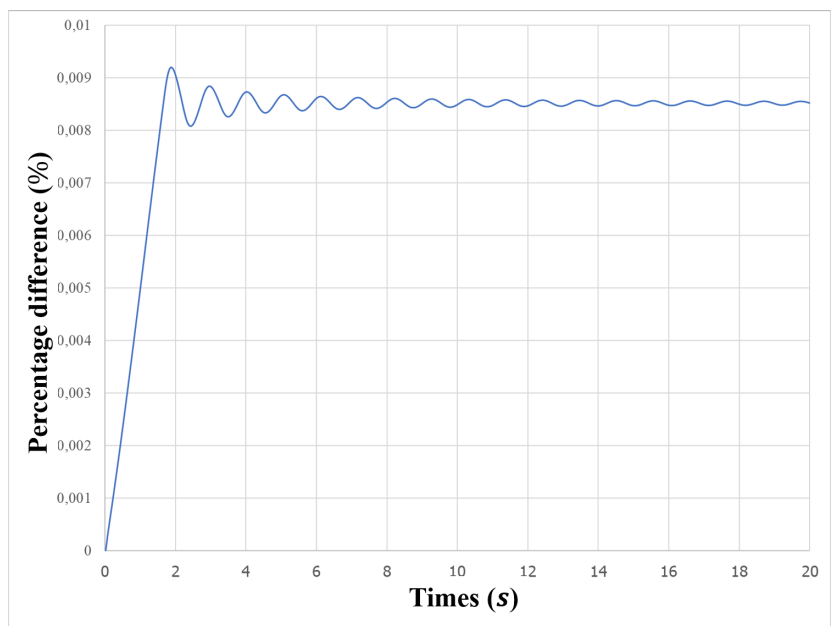

Figure 5. Percentage difference between the piston motion of the complete Modelica model and the counterpart of the simplified model in $\%$ vs. the time.

\section{Numerical Results}

\subsection{Observed behaviours}

To evaluate Sobol's indices, input variables are randomly combined into several sets. Figure 6 shows the displacement time history of the piston for nine sets obtained by combining different values of the input variables. As it can be inferred from these results, semi-periodic (set 2, 3 and 4) or completely damped motions (set 5, 6, 7, 8 and 9) can be obtained by acting on the input variables. In extreme cases, the displacement of the piston is blocked due to a significant value of spring pre-loading (set 1). The displacement time history of the piston obtained with the reference set of parameters of Table 1 is represented by the red curve in Figure 6. The values of the input variables for the other sets are given in Appendix A: for each set, the constant parameters are the same as the reference set (see Table 1).

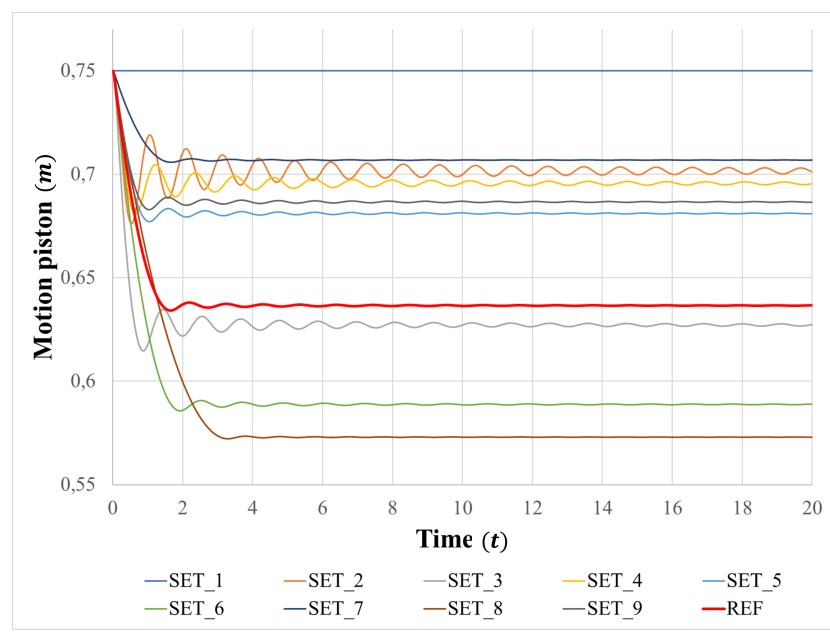

Figure 6. Piston position vs. time for 10 different sets. The red curve is the reference solution.

\subsection{Sensitivity Analysis}

The values of Sobol's indices, have been computed for the considered variables vs. the samples number $N$ for two characteristic time values: $t=\tau_{\mathrm{abs}}$ and $t=10 \tau_{\mathrm{abs}}$.

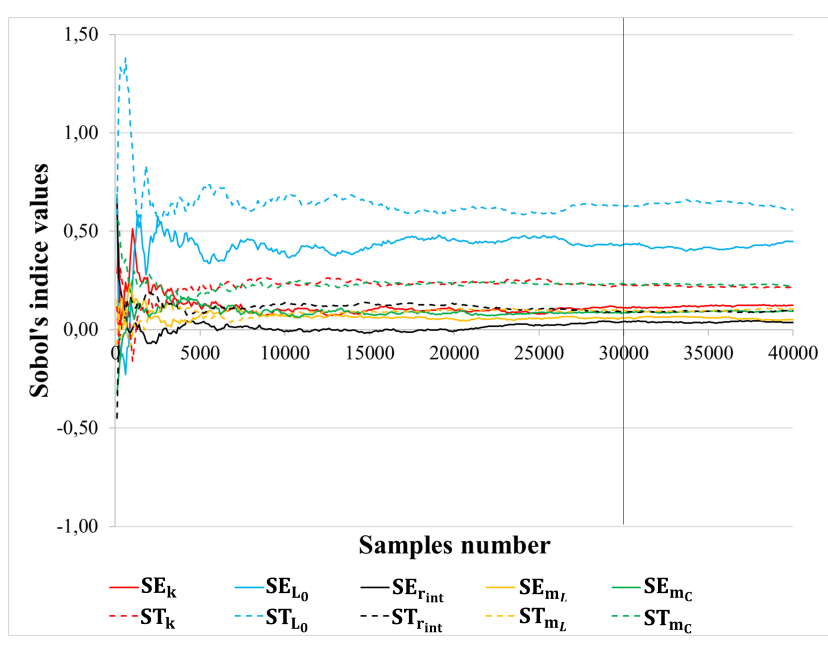

Figure 7. Sobol's indices vs. samples number $N$ for $t=\tau_{\mathrm{abs}}$.

The results of a Sobol's analysis can be considered reliable only if convergence is achieved. The results of Figure 7 and of Figure 8 highlight that for sample numbers lower than 15000 , significant fluctuations exist. These are due to numerical errors, especially for $N<500$ where inconsistent negative values are obtained. When $3000 \leq N \leq$ 15000, another inconsistency is observed since $S E_{L_{0}}$ and $S T_{L_{0}}$ are really not constant. For values of samples number greater than 30000 convergence is achieved for all curves.

As a matter of fact, the standard uncertainty, $\bar{u}$, for the Sobol's indices computed for all the input variables when 


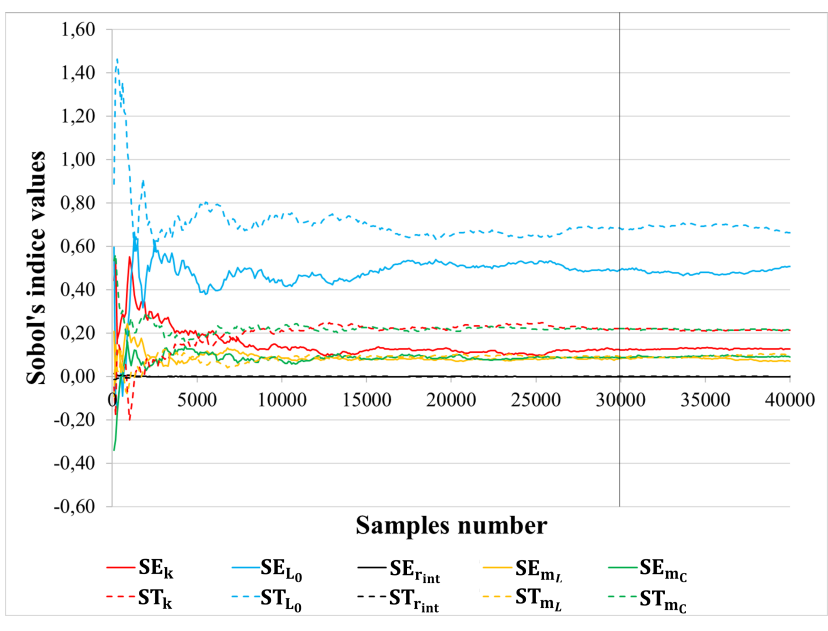

Figure 8. Sobol's indices vs. samples number $N$ for $t=10 \tau_{\text {abs }}$.

the samples number is greater than 30000 is equal to:

$$
\bar{u}=\frac{\bar{\sigma}}{\sqrt{D}}=\frac{5.78 \cdot 10^{-3}}{\sqrt{40000-30000}}=5.78 \cdot 10^{-5},
$$

where $\bar{\sigma}$ is the average standard deviation and $D$ the studied range.

Table 3 reports the values of the converged Sobol's indices at $N=40000$. It must be noticed that, in this table, all values have been multiplied by 100 to enable a better reading.

Table 3. Elementary and total Sobol's indices for $t=\tau_{\mathrm{abs}} \mathrm{s}$ and $10 \tau_{\mathrm{abs}} \mathrm{s}$ in percentage. Note that, by considering their definition in section 2.2, the sum of total indices is not equal to $100 \%$.

\begin{tabular}{|l|l|ll|}
\hline $\begin{array}{l}\text { Total Sobol's in } \\
\text { dex }\end{array}$ & Value at $t=\tau_{\mathrm{abs}}$ & $\begin{array}{l}\text { Value } \\
t=10 \tau_{\mathrm{abs}}\end{array}$ & at \\
\hline ST $K$ & $21.40 \%$ & $21.38 \%$ & \\
ST $L_{0}$ & $60.92 \%$ & $66.24 \%$ & \\
ST $r_{\text {int }}$ & $9.56 \%$ & $0.01 \%$ & \\
ST $m_{P}$ & $10.65 \%$ & $10.29 \%$ & \\
ST $m_{C}$ & $22.66 \%$ & $21.62 \%$ & \\
\hline \hline Elementary & Value at $t=\tau_{\text {abs }}$ & Value & at \\
Sobol's index & & $t=10 \tau_{\text {abs }}$ & \\
\hline SE $K$ & $12.39 \%$ & $12.77 \%$ & \\
SE $L_{0}$ & $44.93 \%$ & $50.85 \%$ & \\
SE $r_{\text {int }}$ & $3.70 \%$ & $0.00 \%$ & \\
SE $m_{P}$ & $4.93 \%$ & $7.16 \%$ & \\
SE $m_{C}$ & $9.32 \%$ & $9.15 \%$ & \\
Interactions & $24.73 \%$ & $20.07 \%$ & \\
\hline
\end{tabular}

A total Sobol's index can help understanding the influence of a given input variable on the piston motion when combined with other variables. For instance, in the case of the total index of $m_{P}$ at $t=\tau_{\mathrm{abs}} \mathrm{s}$, its value is $10.65 \%$, which is at least two times smaller than the other ones, like $r_{\text {int }}$.

As far as variable $L_{0}$ is concerned, always at $t=$ $\tau_{\text {abs }} \mathrm{s}$, a significant difference exists between its elemen- tary Sobol's index, $44.93 \%$, and the total one, $60.92 \%$. This means that this parameter plays a crucial role on the piston motion in combination with other parameters.

Finally, by prioritising the other indices, it can be concluded that the parameter $L_{0}$ is the one influencing the most the motion of the piston.

The same remarks can be repeated for $t=10 \tau_{\mathrm{abs}}$, except for $r_{\text {int }}$ whose elementary and total Sobol's indices become smaller than the ones related to the other input variables. This is due to the fact that $r_{\text {int }}$ is involved only in the expression of the damper force. This force being function of the piston speed, it is possible to link indirectly $r_{\text {int }}$ with the others parameters. Thus, when the system reaches a stable state, the piston velocity goes to zero and the influence of parameter $r_{\text {int }}$ too.

The results of Table 3 also highlight that the parameters $k, m_{P}$ and $m_{C}$ present similar values of the total Sobol's index at both time, $t=\tau_{\mathrm{abs}} \mathrm{s}$ and $t=10 \tau_{\mathrm{abs}} \mathrm{s}$. Nonetheless, if a greater range of $r_{\text {int }}$ is considered, the influence of this parameter on the motion regime can be observed. Indeed, for values of $r_{\text {int }}$ greater than $0.005 \mathrm{~m}$, the motion of the piston is mainly semi-periodic or completely periodic without stable motion. This highlights the importance of correctly defining the interval of the input variables to be used during the sensitivity analysis. Furthermore, inasmuch as $r_{\text {int }}$ is the input variable with the weakest influence on the piston motion, its value can be set randomly without influencing the final result. Conversely, particular care must be put in choosing the value and the interval of definition of the most influencing variables (like $L_{0}$ ).

The coupling of the FMU file of the car shock absorber Modelica model with the Nebraska code allows carrying out for fast simulations to be carried out. In fact, the Modelica model, shown in Figure 4, requires approximately $4.50 \mathrm{~s}$ to run while the sensitivity analysis with Nebraska (which makes use of the FMU file generated from MapleSim) takes approximately $5000 \mathrm{~s}$ for a total of 280000 simulations. All the simulation presented in this paper have been carried out on a DELL ${ }^{\circledR}$ laptop with $32 \mathrm{~Gb}$ RAM and an Intel core i7-10610 processor. From the analysis of the time required to execute each step of the simulation, one can conclude that about $50 \%$ of the overall time is required to solve (and integrate) the problem, while the remaining $50 \%$ of the time is needed for post-processing of results. In particular, the time required to execute each step is:

\section{Equations generation $(1.131 \mathrm{~s})$;}

2. Equations handling and manipulation (0.219 s);

3. Initial values computation (0.224 s);

4. Integration step $(0.710 \mathrm{~s})$;

5. Post processing of results (2.216 s). 


\section{Conclusion}

In this work a sensitivity analysis of a car shock absorber model, carried out through the coupling of an in-house code (Nebraska) based on Sobol's indices and a FMU file generated via MapleSim, has been presented.

The goal is to identify a reduced model capable of describing the motion of the piston. To this purpose, the influence of the different physical phenomena on the considered output has been investigated. The system, described via several geometrical and mechanical parameters, has been firstly analysed by observing the order of magnitude of the different forces acting on the system. As a first result, the initial model has been simplified by removing those forces whose mechanical effects are negligible when compared to others.

The simplified model was then used to generate the FMU file, which was coupled with the based-Fortran code Nebraska through a Python code (in-house developed) which carried out the sensitivity analysis via Sobol's indices.

The analysis of both the elementary and total Sobol's indices of the input parameters of the mechanical system allowed determining that the spring unstreched length $\left(L_{0}\right)$ plays a crucial roles in the piston motion while the influence of $r_{\text {int }}$ is negligible. Note that this result holds solely for the output considered in this study, i.e. the position of the piston when a stable state is reached.

This study represents a first application of FMU to simulate mechanical systems with the aim of performing sensitivity analysis. A further extension of the approach presented in this work could be its application to more complex systems. In this context, the more influential subsystems, determined as a result of a sensitivity analysis based on Sobol's indices, or subsystems involving complex physical phenomena that must be accurately reproduced, could be replaced by a external model imported via a FMU file. As far as prospects of this work are concerned, the FMU can be generated from dedicated finite element models or meta-models, integrating the relevant physical responses. This can help reducing the computational cost of the sub-system simulation required within the global model of the mechanical system developed in MapleSim.

\section{Acknowledgements}

The authors would like to thank Laurent JACQUI who developed the Nebraska code.

\section{References}

Chastaing, G., F. Gamboa, and C. Prieur (2015). “Generalized Sobol sensitivity indices for dependent variables: numerical methods". In: Journal of Statistical Computation and Simulation 85.7, pp. 1306-1333. DOI: 10.1080/00949655.2014. 960415

Fritzson, Peter (2014). Principles of Object Oriented Modeling and Simulation with Modelica 3.3: A CyberPhysical Approach. ISBN: 9781118989166. DOI: 10.1002/ 9781118989166.

Héliot, Maxime (2017-2018). "Studies and development of a sensitivity analysis tool and application ti an aerothermal model". MA thesis. Cranfiel University.

Hoeffding, Wassily (1948). "A Class of Statistics with Asymptotically Normal Distribution”. In: Annals of Mathematical Statistics 19.3, pp. 293-325.

Idel'chik, I.E. (1966). Handbook of hydraulic resistance. Ed. by IPST Staff D. Grunaer P.E. The U.S. Atomic Energy Commission and The National Science Foundation.

Janon, Alexandre (2019-06). "Global optimization using Sobol indices". working paper or preprint. URL: https : / / hal . archives-ouvertes.fr/hal-02154121.

Maplesoft, Copyright (2014). MapleSim User's Guide. ISBN: 9781926902326.

Marrel, Amandine et al. (2009). "Calculations of Sobol indices for the Gaussian process metamodel". In: Reliability Engineering and System Safety 94.3, pp. 742-751.

Philippe, Anne and Marie-Claude Viano (2010). "Cours de probabilités : Modèles et Applications".

Plogert, Klaus (1996). "The tailoring process in the German VModel". In: Journal of Systems Architecture 42, pp. 601-609.

Saltelli, Andrea (2002). "Making best use of model evaluation to compute sensitivity indices". In: Computer Physics Communications 145.(2), pp. 280-297.

Sobol, I.M. (1993). "Sensitivity Estimates for Nonlinear Mathemitical Models". In: MMCE 1.4, pp. 407-414.

\section{Acronyms}

$\begin{array}{ll}\text { MBSE } & \text { Model-Based System Engineering } \\ \text { MISO } & \text { Multiple-Input-Single-Output } \\ \text { SE } & \text { Sobol Elementary Index } \\ \text { ST } & \text { Sobol Total Index } \\ \text { FMU } & \text { Functional Mock-up Units } \\ \text { PDF } & \text { Probability Density Function } \\ \text { CAD } & \text { Computer Aided Design } \\ \text { MSL } & \text { Modelica Standard Library }\end{array}$




\section{Glossary}

$N \quad$ Input variables sets number $\quad-$

$d \quad$ Number of input variables -

$\zeta \quad$ Proportional factor in singularity -

$\tau_{\text {abs }} \quad$ Absorbing motion time

$\Delta P \quad$ Pressure loss $\quad \mathrm{Pa}$

$k \quad$ Spring constant $\quad$ N.m ${ }^{-1}$

$L_{0} \quad$ Spring unstreched length $\quad \mathrm{m}$

$r_{\text {int }}$ Internal piston radius $\mathrm{m}$

$r_{\text {ext }} \quad$ External piston radius $\quad \mathrm{m}$

$h \quad$ Piston thickness $\mathrm{m}$

$x_{\text {start }} \quad$ Piston initial position $\quad \mathrm{m}$

$L_{\text {cham }} \quad$ Length of the piston chamber $\mathrm{m}$

$L_{\text {bar }} \quad$ Length of the bar linked to the $m$

$\omega_{0} \quad$ Fluid speed $\quad \mathrm{m} . \mathrm{s}^{-1}$

$g \quad$ Gravity acceleration $\mathrm{m} . \mathrm{s}^{-2}$

$S_{p} \quad$ Piston surface $\quad \mathrm{m}^{2}$

$S_{h} \quad$ Piston hole surface $\quad \mathrm{m}^{2}$

$m_{P} \quad$ Passengers mass $\quad \mathrm{kg}$

$m_{C} \quad$ Car and passengers mass $\quad \mathrm{kg}$

$\rho_{\text {oil }} \quad$ Oil density $\quad$ kg.m ${ }^{-3}$

$\bar{u} \quad$ Mean of standard uncertainly -

$\bar{\sigma} \quad$ Mean of the average standard de- viation

\section{Appendix A}

Table 4. Variable parameters data for ten different sets.

\begin{tabular}{|l|l|l|l|l|l|}
\hline Parameter & set 1 & set 2 & set 3 & set 4 & set 5 \\
\hline$K \mathrm{~N}^{-1} \mathrm{~m}^{-1}$ & 10768 & 10935 & 9513 & 9811 & 9830 \\
$L_{0} \mathrm{~m}$ & 0.585 & 0.498 & 0.475 & 0.562 & 0.480 \\
$r_{\text {int }}\left(10^{-3} \mathrm{~m}\right)$ & 3.63 & 4.20 & 3.24 & 3.37 & 2.49 \\
$m_{P} \mathrm{~kg}$ & 239 & 140 & 178 & 213 & 111 \\
$m_{C} \mathrm{~kg}$ & 964 & 1182 & 1172 & 1252 & 1086 \\
\hline \hline Parameter & set 6 & set 7 & set 8 & set 9 & ref \\
\hline$K \mathrm{~N}^{-1} \mathrm{~m}^{-1}$ & 9329 & 8951 & 9306 & 10345 & 9000 \\
$L_{0} \mathrm{~m}$ & 0.479 & 0.561 & 0.407 & 0.533 & 0.450 \\
$r_{\text {int }}\left(10^{-3} \mathrm{~m}\right)$ & 2.25 & 1.77 & 1.75 & 2.42 & 2.25 \\
$m_{P} \mathrm{~kg}$ & 196 & 135 & 359 & 310 & 150 \\
$m_{C} \mathrm{~kg}$ & 1287 & 1156 & 908 & 1151 & 1000 \\
\hline
\end{tabular}

